\title{
Una propuesta didáctica para la enseñanza de los conceptos estructurantes de discontinuidad de la materia y unión química desde la epistemología y la historia de la ciencia contemporáneas
}

Carlos J. Mosquera S. Leidy G. Ariza A. Andrea M. Reyes G. Carlos Hernández R. *

\section{RESUMEN}

Esta propuesta didáctica está orientada, mediante el uso de conceptos estructurantes de la naturaleza corpuscular de la materia y del enlace químico, a la enseñanza de la estructura atómica y su relación con las propiedades macroscópicas que presenta la materia en los estados sólido, líquido y gaseoso. Así mismo, su construcción es producto de un análisis teórico de antecedentes referidos a diversos aspectos: historia de la química, epistemología de las ciencias, modelo pedagógico constructivista, modelo didáctico por resolución de problemas, psicología cognitiva y relaciones $\mathrm{CTS}^{1}$. Por su parte, la unidad didáctica está conformada por siete actividades en las que se incluye la comprensión de las ideas previas de los estudiantes, cinco ejercicios de enseñanza-aprendizaje y un examen final para evaluar el alcance de competencias a lo largo de la aplicación del proyecto.

\section{Palabras clave:}

Concepto estructurante, historia de la ciencia, enseñanza y aprendizaje de la ciencia, resolución de problemas.

\section{SUMMARY}

This didactic proposal is oriented towards the educational problems about the relationship between atomic structure and macroscopic properties that shows matter in the solid, liquid and gas states, using structural con-

\footnotetext{
* Grupo de Investigación de Didáctica de la Química “Didaquim”, Universidad Distrital Francisco José de Caldas - cmosquera@udistrital.edu.co.

1 Relaciones Ciencia, Tecnología y Sociedad: línea de investigación profusamente abordada desde comienzos de la década del noventa en el campo de conocimiento de la Didáctica de las Ciencias Experimentales.
} 
cepts of corpuscular nature of the matter and chemical bond. It's construction is product of a theoretical analysis of antecedents referred to diverse aspects such as: history of chemistry, science epistemology, constructivism, problem solving didactic model, cognitive psychology and relationships STS, it is presented in a didactic unit made up of seven activities, including the previous knowledge analysis of the students, five teaching-learning activities and a final activity in which the reach of skills is evaluated along the application of the proposal.

\section{Keywords:}

Structural concept, history of science, science education and learning, problem solving.

\section{Introducción}

Elaborar una unidad didáctica para la enseñanza de la química, basada en el modelo por resolución de problemas, no es una idea del todo innovadora. De hecho, la creación de unidades didácticas cuenta con un número creciente de referencias sobre experiencias pedagógicas y evaluaciones de los principios que rigen la planificación de las mismas. La didáctica de las ciencias, en tanto disciplina de conocimiento en el contexto de las ciencias de la educación, da indicios de un desarrollo evolutivo de un conjunto de reglas implícitas que tiene la comunidad académica para hacer público sus saberes (Aduriz \& Izquierdo, 2002) y relacionarlos con el abordaje y la propuesta de solución de problemas referentes a la enseñanza y el aprendizaje de las ciencias (Gil, 2000). En la actualidad existe un interés marcado por diferenciar las relaciones entre conocimiento científico, conocimiento cotidiano y conocimiento escolar. Éstas nociones son integradas por los docentes a través de estrategias de enseñanza, las cuales buscan facilitar la adquisición constructiva del conocimiento científico escolar y desarrollar habilidades y destrezas de pensamiento y acción - junto con el desarrollo de la dimensión humana de las personas valoradas como seres sociales - para configurar la noción de lo público en la cultura. Hacia éstas finalidades está orientada la propuesta de unidad didáctica.

La unidad didáctica titulada ¿Qué hay en común entre un sólido, un líquido y un gas?, tiene como propósito articular ciertos conceptos estructurantes de la química, como la naturaleza corpuscular de la materia y la unión química, desde un enfoque histórico y epistemológico y, además, formar una estructura apropiada para ser desarrollada en un currículo de ciencias propuesto para los grados décimo y undécimo del sistema educativo colombiano (especificado en los estándares de competencias del MEN). Dicha articulación pretende enfocar el proceso enseñanza-aprendizaje de la química - en los campos conceptual, procedimental y actitudinal — hacia el desarrollo de competencias científicas escolares.

El proyecto, fundamentado en principios que buscan atender las demandas exigidas por la dinámica de la sociedad, se orienta en metodologías propias de la investigación contemporánea acerca de la formación de las ciencias y en alternativas para abordar la evolución de los procesos de enseñanza-aprendizaje —iniciada por Novak en 1988. En otras palabras, el objetivo de ésta propuesta es contribuir al desarrollo de la enseñanza de las ciencias y posibilitar nuevos campos de discusión acerca de los modelos didácticos.

En el marco de la epistemología de las ciencias existe, sin lugar a dudas, un amplio número de propuestas para sustentar los cambios del conocimiento científico (en función del tiempo) y de las teorías que intentan explicar los fenómenos naturales y/o sociales. Por lo tanto, la historia, junto con el estudio mismo de las ciencias, se constituye en un factor relevante en la investigación didáctica. Es aquí donde se resalta la importancia de la historia de las ciencias para ofrecer validez a los modelos explicativos de la evolución científica.

El trabajo se fundamentó en el diseño y la realización de unidades didácticas bajo el referente conceptual-epistemológico del modelo de Dinámica Científica, propuesto por Anna Estany (1990), y en 
la epistemología social que expone Gerard Fourez (1994). Mediante el acercamiento de la historia de la ciencia $-y$ de posturas crítico sociales sobre su trabajo en el aula- se buscó evidenciar la mejora del aprendizaje de contenidos conceptuales, procedimentales y actitudinales en los estudiantes.

A partir de estos principios, teniendo en cuenta la caracterización de actitudes en los estudiantes y la conceptualización de los contenidos, se elaboró una unidad didáctica orientada al desarrollo de conceptos estructurantes como unión química y naturaleza corpuscular de la materia y de vacío. Lo anterior tomando como base una perspectiva histórica proporcionada por la articulación de las apreciaciones epistemológicas citadas y algunos aportes de investigaciones referentes a los campos mencionados. De hecho, la unidad didáctica enlaza dichos conceptos estructurantes con el desarrollo de actitudes hacia la ciencia y su implementación práctica en el aula (abordando casos de la vida cotidiana de los estudiantes).

\section{¿Qué bases didácticas y cognitivas son necesarias para validar la propuesta de enseñanza y aprendizaje de las ciencias?}

En las clases tradicionales de ciencias, los estudiantes solucionan problemas planteados a partir de la aplicación de una ecuación indicada; sin embargo, el aprendizaje es básicamente memorístico, aleatorio y difícil de trasponer a situaciones que impliquen un tipo de análisis distinto o que no contengan las variables determinadas para aplicar el algoritmo. En consecuencia, este mecanismo generará frustración y apatía en el estudiante cuando intente resolver un cuestionamiento nuevo.

Por su parte, el aprendizaje significativo propuesto por Ausubel, Novak y Hanessian (1976), centrado en el conocimiento y en la modificación de la estructura cognitiva del estudiante, propone "anclar" nuevos conceptos que le permitan representar la realidad categórica y esquemáticamente simplificada (relacionando, reorganizando y asimilando significados modernos que le permitan manipular y comprender su realidad). La resolución de problemas contribuye a que el estudiante construya esas bases y asimile los problemas de química como problemas abiertos y susceptibles de ser abordados a través de estrategias guiadas por sus propias hipótesis, objetivos e intereses - justificado desde un marco teórico coherente. Ésta metodología incrementa la motivación por las clases de ciencias; los estudiantes son los que construyen su propio conocimiento e incluso pueden solucionar problemas planteados por ellos mismos. El desarrollo de este tipo de actividades le otorga confianza al estudiante para desenvolverse en diferentes tipos de competencias y así enfrentarse a ejercicios de disciplinas diversas.

Consecuente con lo anterior, uno de los objetivos de la unidad didáctica es la interiorización significativa de los conceptos estructurantes en el discurso escolar del estudiantado, pues para la comprensión de éste trabajo es indispensable esclarecer la diferencia entre lo que se entiende por concepto ("concepto ausubeliano") y por conceptos didácti$\cos$ (concepto estructurante). Éste último, en términos psicológicos, puede interpretarse como un término inclusivo en el que se consideran varias ideas preconcebidas en la mente del estudiante; abarca todos los atributos de criterio relacionados a eventos, objetos, fenómenos y situaciones que se mezclan para dar forma, mediante su enseñanza, a la estructura cognitiva de los estudiantes.

Además, el concepto estructurante tiene una naturaleza independiente en comparación a los ausubelianos ya que, de acuerdo con su misma existencia y características, el problema de su enseñanzaaprendizaje es diferente. De esta manera se asume que la noción de concepto didáctico ${ }^{2}$ como $<<$ naturaleza corpuscular de la materia $>>,<<$ cambio químico $>>,<<$ cuantificación de relaciones en química $>>$ y $<<$ equilibrio químico $>>$, en la mayoría de los casos -particularmente en la enseñanza de la química- se presenta en forma de proposiciones. No obstante, también se distinguen algunos conceptos muy inclusivos que, al parecer, son posibles de encontrar con alto grado de jerarquía en la estructura cognoscitiva de los alumnos como pro-

2 Denominados estructurantes en la literatura didáctica.

REVISTA CIENTÍFICA / ENERO -DICIEMBRE DE 2010 / No. 12 / BOGOTÁ, D.C. 
ducto de sus aprendizajes (de corte ausubeliano: entropía, energía, varianza, célula, etc.).

Los individuos, con la adquisición del concepto didáctico, no otorgan el mismo significado genérico a los términos de su lenguaje; éstos organizan su discurso de tal manera que, al mitigar la influencia de los elementos idiosincrásicos sobre las definiciones, son organizados (estructurados) por la significatividad de las relaciones entre ellos mismos. Entonces, la jerarquización entre las relaciones y los conceptos está supeditada por la existencia del concepto didáctico (estructurante) adquirido durante el proceso de enseñanza-aprendizaje de la ciencia.

¿Cómo integrar la historia de la química en la propuesta didáctica? El papel de la historia en diferentes campos de la actividad científica, sobre todo en la didáctica, es tan relevante como lo afirma Quintanilla (2005):

Promueve una mejor comprensión de los conceptos y métodos científicos; los enfoques históricos conectan el desarrollo del pensamiento individual con el desarrollo de las ideas científicas; la historia de la ciencia se hace necesaria para comprender la naturaleza de la ciencia, su objeto y su método de estudio; la historia de la ciencia cuestiona el cientificismo y dogmatismo que es común de encontrar en nuestras clases y nuestros textos de ciencia.

En consecuencia, la historia no debe ser sólo un bloque temático en los currículos de ciencias, una estrategia para enseñar hechos y datos o un capítulo introductorio; más bien, debe ser empleada desde una perspectiva constructivista y por reconstrucción racional, un factor determinante como elemento didáctico. La historia de la ciencia, en los procesos de enseñanza-aprendizaje, favorece el establecimiento de actitudes positivas de los estudiantes hacia la asignatura; propicia cambios frente a la visión que tienen acerca de la manera en la que los científicos resuelven sus conflictos. ${ }^{3}$. En tal sentido, como fundamento de esta investigación,

3 Problemas cuyo origen está delimitado por una época, una sociedad y unos elementos culturales. han surgido los siguientes interrogantes: ¿Cómo organizar y estructurar los cambios en conceptos estructurantes dentro de un campo de conocimiento? ¿cuál es el papel de la historia de la ciencia para construir una unidad didáctica dirigida a la enseñanza de conceptos estructurantes en perspectiva dinámica y cambiante? Para dar respuesta a estas preguntas fue empelado, como herramienta principal, el modelo de Dinámica Científica propuesto por Anna Estany; no pretende analizar los cambios experimentados por las teorías científicas sino enfocarse en el estudio de un fenómeno más complejo: la evolución de las ciencias.

El enfoque de esta propuesta radica en revelar problemáticas de actitudes negativas en los estudiantes de secundaria hacia el aprendizaje de las ciencias (Ariza y Currea, 2004). A causa de los métodos tradicionales de enseñanza, se observan dificultades en la construcción significativa de conceptos en química como, por ejemplo, las interacciones que hacen parte del modelo corpuscular de la materia (fuerzas "intra" e "intermoleculares" -conceptual-), la metodología y las actividades propias del trabajo en ciencias (observación, toma de datos $\mathrm{u}$ organización de la información -procedimental-) y las actitudes con las que se enfrentan los estudiantes a las actividades de la propuesta (motivación, rigurosidad y planteamiento de propuestas verificables -actitudinal-). Por esta razón surgió la necesidad de diseñar una unidad didáctica que afrontara éstas dificultades y posibilitara la estructuración de una visión de ciencia de acuerdo a los principios epistemológicos actuales.

Abordar dicha temática implicó la contextualización en torno a dos conceptos estructurantes, la unión química y la naturaleza corpuscular de la materia. Así mismo, las actividades fueron planeadas con base en el modelo de resolución de problemas y algunos referentes históricos, epistemológicos, didácticos y pedagógicos. De tal forma, los fundamentos interdisciplinarios de la unidad favorecen en los estudiantes el desarrollo con una visión dinámica de la construcción de ciencia ${ }^{4} \mathrm{y}$

4 Asumiendo de forma implícita que la construcción de ciencia se explica desde diferentes aspectos conceptuales, actitudinales, procedimentales y sociales. 
permiten hacer explícitas las interacciones entre los elementos sobre los cuales se teoriza.

La perspectiva de este estudio sugiere el aprendizaje como una construcción consciente que establece cambios fuertes y débiles entre representaciones, concepciones, actitudes y esquemas de acción. En ese sentido, la unidad elaborada propone la generación de cambios conceptuales, procedimentales $\mathrm{y}$ actitudinales en los alumnos mediante el modelo de enseñanza por resolución de problemas y así, integrando desarrollos epistemológicos de la ciencia y la historia de la química, poder abordar los conceptos de unión química y naturaleza corpuscular de la materia. Lo anterior como estrategia para fomentar el desarrollo de competencias científicas en los estudiantes.

\section{Estrategia metodológica.}

El procedimiento utilizado fue de corte interpretativo-descriptivo y longitudinal en el tiempo; es decir, el tratamiento de la muestra se ejecutó desde un tiempo inicial $t_{1}$ hasta un tiempo final $t_{2}$, (compilando información a lo largo del proceso de enseñanza a través de los instrumentos propuestos en la unidad). Se construyó, entonces, un diseño metodológico coherente basado en los siguientes supuestos:

1. La posibilidad de generar, en los estudiantes, una visión dinámica de la construcción y evolución del conocimiento científico considerando las interacciones de las ciencias (si su enseñanza es llevada a cabo mediante unidades didácticas desarrolladas en el marco de la producción actual de la epistemología y la historia de las ciencias).

2. Mediante la aplicación de las actividades de enseñanza dentro de la unidad didáctica propuesta, los estudiantes experimentarán cambios en los campos conceptual, procedimental y actitudinal - frente a la visión de ciencia y especialmente - con respecto a los conceptos estructurantes de unión química y naturaleza corpuscular de la materia.
3. Luego del desarrollo de la unidad didáctica, los estudiantes serán capaces de resolver problemas cotidianos, científicos y escolares utilizando como referente teórico los conceptos estructurantes de naturaleza corpuscular de la materia y unión química.

La unidad didáctica se aplicó a 28 estudiantes, entre los 15 y 18 años, de educación secundaria ${ }^{5}$ en el contexto académico de la asignatura de química. El diseño de la actividad $\mathrm{N}^{\circ} 0$, De concepciones alternativas, está encaminado a proveer situaciones contextualizadas en el entorno cotidiano de los alumnos sin implicar su influencia sobre la manera en que se unen las partículas para formar enlaces químicos tras una reacción (la exploración busca encontrar la diferenciación que hacen los estudiantes entre el nivel macroscópico de la materia y el nivel microscópico).

Las ideas previas de los estudiantes, con el propósito de identificar en qué estado se encuentran y así determinar qué estrategias llevar a cabo en la siguiente aplicación de las actividades, son analizadas en los ambientes: a) conceptual, a partir de los pictogramas propuestos para indicar conceptos básicos como vacío, molécula, enlace, átomo y reacción; b) procedimental, mediante la formulación de preguntas, hipótesis y registro de observaciones; y c) actitudinal, enfocado hacia la ciencia, su aprendizaje y las implicaciones sociales de conocimiento científico.

En seguida se efectúa un seguimiento de los cambios cognitivos desarrollados a lo largo de las actividades 1 a 6 de la siguiente manera: en lo conceptual se analiza la intervención de los estudiantes mediante mapas conceptuales prediseñados en la propuesta y construidos por ellos mismos. En ellos deben evaluarse los niveles de jerarquía de diferentes conceptos, proposiciones formadas, conexiones cruzadas y ejemplos (Novak, J. y Gowin, D., 1988). Por su parte, en el campo procedimental se indagará acerca de la forma en que los estudiantes resuelven problemas tanto de lápiz y papel abiertos como transforma-

5 Colegio de carácter privado con sede en Bogotá D.C.

REVISTA CIENTÍFICA / ENERO -DICIEMBRE DE 2010 / No. 12 / BOGOTÁ, D.C. 
dos ; $\mathrm{y}$, finalmente, lo referente a lo actitudinal se valorará a través de los diarios de campo y de tareas anexas a las actividades que den cuenta de éste tipo de aspectos.

El seguimiento de la actividad final se presenta en términos de los saberes procedimentales, conceptuales, actitudinales y de las competencias alcanzadas desde el test de ideas previas hasta la finalización de la unidad didáctica. Además, se exhibe una variación en el análisis de los saberes actitudinales, pues se identifican con el uso de escalas Likert en los últimos cinco ítems de la actividad.

Durante el desarrollo de las actividades 1 a 6 , se abarcan los contenidos de enseñanza propuestos por la trama conceptual (conformada por los anexos 1 y 2) creada a partir de los estándares de competencias dispuestos en el esquema histórico y conceptual. Las actividades se presentan siguiendo el orden establecido por la trama conceptual (anexo 1 y 2) en lo referente al elemento histórico (una red de preguntas cuya resolución es objeto de estudio en cada una de las actividades y un mapa histórico que contiene los conceptos jerárquicamente incluidos en las actividades). Inicialmente se estudia el estado gaseoso, seguido por el líquido y finalmente el sólido, todo dirigido por un hilo conductor preestablecido en las unidades básicas fundamentadas a partir el desarrollo histórico de la ciencia que comprenden desde el atomismo griego hasta los modelos atómicos precuánticos.

\section{Conclusiones}

Antes de la aplicación de la unidad, en términos generales, los estudiantes poseían fuertes obstáculos conceptuales para determinar la naturaleza discontinua de la materia; sus ideas, en la mayoría de las situaciones, eran contradictorias ya que oscilaban entre aspectos corpusculares y continuos. Sobre la transformación química se analizaron interpretaciones simbólicas y, en algunos casos, otras no muy consistentes aprendidas en la experiencia escolar, hecho que nos hace suponer que, al trans- poner éstas ideas al mundo real, pueden implicar dificultades para admitir la situación de la reacción química o la existencia de vacío entre partículas.

Entre tanto, los criterios propuestos son un aspecto a considerar; tan sólo en uno caso se presentó el uso de más de un criterio para referirse a la aceptación de una teoría por encima de otra. Lo anterior da cuenta del pensamiento unidimensional con el que cuentan los estudiantes y, por lo tanto, se asume que con la introducción del modelo epistemológico de Anna Estany es posible la restructuración de las ideas sobre los aspectos metodológicos.

Encuanto ala estructura - netamente conceptualconcordamos con los investigadores Pozo (1991 y 1999), Benarroch (2000) y de Posada (1999); los términos químicos son aprendidos por recepción y memorísticamente. Lo anterior se evidencia en los resultados obtenidos donde los estudiantes manifiestan, en sus respuestas, la relación con los modelos tradicionales (gráficas especialmente) que aparecen en los libros de texto. Como sustento de ésta noción se encuentra el esquema conceptual presentado en la actividad de concepciones previas, pues todos los alumnos relacionaron causalmente los conceptos electrón, protón y electrón (¿neutrón?) subordinados al núcleo atómico. Entonces, la integración de éstos conceptos a la estructura cognitiva no resulta del todo fructífera para el discurso científico escolar pero sí es efectiva como argumentación memorística para dirigirse al profesor.

Sobre los criterios actitudinales, en el inicio de la unidad didáctica, los resultados no fueron sorpresivos si se tiene en cuenta la trayectoria tradicional con la que han sido formados los estudiantes en las ciencias. De hecho, manifiestan ideas de su naturaleza, su construcción, su aprendizaje y la percepción que tienen del científico — compatible con imaginarios ahistóricos y descontextualizados. Así mismo, se puso en evidencia que en cinco de los catorce casos observados existe una correlación entre las ideas de la construcción de ciencia - por medio de la investigación - para satisfacer fines cognitivos del investigador; siendo éste último criterio el más utilizado por los estudiantes. 
A través de un análisis más detenido fue posible determinar que los estudiantes se encuentran inicialmente en el tercer nivel explicativo, entendiendo éste como un elemento heurístico - propuesto por Alicia Benarroch (2000) - para explicar, en términos generales, la estructura cognoscitiva de los estudiantes mediante sus respuestas. Éstas se clasifican en cinco categorías que dan cuenta del grado de pertinencia empleado por los alumnos para resolver situaciones en las que entran en juego la naturaleza discreta de la materia con algunas excepciones que dan cuenta de los niveles II y IV. Según Benarroch, se catalogan en:

Nivel I: los alumnos conciben la materia como continua y estática. Además, no consideran necesaria la explicación de los cambios físicos y químicos de la materia.

Nivel II: las sustancias se conciben como "embutidos de partículas" (éstas son vistas desde una perspectiva microscópica, también, como en el nivel I). Incluso, los estudiantes exponen la relevancia de explicar las situaciones propuestas en los instrumentos.

Nivel III: en las respuestas, los alumnos perciben escalas corpusculares para identificar los cambios de la materia (no se concibe la necesidad del vacío).

Nivel IV: los alumnos comprenden la materia - compuesta de partículas y vacío, principalmente - en donde la naturaleza de las sustancias está determinada por la ausencia o presencia de la cantidad de vacío según la disposición de la misma.

Nivel V: éste último esquema coincide con las explicaciones corpusculares en las que entran en juego las interacciones entre las partículas por medio de fuerzas, tanto ínter como intramoleculares, y su movimiento intrínseco.

En cuanto al campo epistemológico y procedimental, es posible asegurar la existencia de diversas ideas en lo que respecta al cambio de teorías; son evidentes las representaciones concernientes a la caracterización de la evolución empirista de la ciencia.

Una vez aplicada la unidad didáctica fueron notables algunas diferencias en el objeto de estudio; los cambios experimentados por los estudiantes estuvieron propiciados por la aplicación de una actividad pedagógica construida a partir de referentes dinámicos, históricos y epistemológicos contemporáneos.

Al término de la unidad, los estudiantes emplearon conceptos explicativos cercanos a los de la naturaleza corpuscular de la materia y la unión química para exponer la interacción entre partículas y, a pesar que en algunas oportunidades no fueron utilizados de manera adecuada, demostraron la necesidad de incluirlos en sus discursos. Según sus respuestas, los alumnos se catalogaron en el nivel III explicativo (con algunas excepciones ubicadas en el IV). Empero, evidenciaron un desarrollo notable con relación a los resultados iniciales; se hicieron notorias algunas escalas corpusculares de interacción para identificar los cambios de la materia sin la necesidad del vacío. Luego de éste avance, la actividad puede considerarse como un cambio conceptual apreciable.

Los siguientes fueron los conceptos reafirmados en la estructura cognitiva de los estudiantes: interacción, molécula, átomo, mezcla, concentración, disolución, sustancia, elemento y compuesto. Como novedad concluimos que dichos términos son abordados ahora desde un enfoque corpuscular interactivo.

A medida que los estudiantes se enfrentaron con problemas no formulados de modo habitual - cuantitativamente - sino como asuntos abiertos y cualitativos, ${ }^{6}$ fue notable una mejora progresiva y homogénea en cuanto a la delimitación de los problemas. Lo anterior es una consecuencia del carácter abierto de las situaciones que supera la asignación de valores que, de alguna manera, son estándar para el desarrollo del componente algorítmico de la resolución.

6 Cabe anotar que se les propuso, a partir de enunciados, los criterios procedimentales a tener el cuenta en el momento de planificar una estrategia de resolución.

REVISTA CIENTÍFICA / ENERO -DICIEMBRE DE 2010 / No. 12 / BOGOTÁ, D.C. 
Con base en los resultados obtenidos en cada una de las repuestas y luego de analizar su percepción con respecto a la construcción de la ciencia, es posible afirmar que los alumnos la infieren en dos enfoques: las revoluciones científicas y los modelos de dinámica científica. No obstante, aunque se generaron actitudes positivas, el trabajo con este tipo de enseñanza debe ser más extenso para alcanzar todos los logros propuestos y generar cambios radicales en las concepciones del estudiantado - lo cual es bastante plausible; si en sólo tres semanas (tiempo de duración de la aplicación de la unidad) se consiguieron avances importantes, con un trabajo constante se podrá modificar significativamente la estructura cognitiva de los estudiantes.

Acerca de sus actitudes - en cuanto a las implicaciones sociales de la ciencia- puede concluirse que, debido a la introducción de los referentes históricos, la mayoría de los alumnos, después de la experiencia de ésta unidad didáctica, demuestran predisposiciones hacia actitudes favorables con los presupuestos epistemológicos aceptados en el marco conceptual de este trabajo; es decir, admiten la construcción de la ciencia como una labor social regulada por los criterios racionales de los investigadores. Finalmente, los estudiantes reconocen en su discurso las relaciones entre la ciencia y la tecnología y sus implicaciones en la sociedad actual.

Como conclusión general se determina que una unidad didáctica de orientación constructivista, apoyada por los desarrollos históricos y epistemológicos de las ciencias, y enfocada en un modelo de enseñanza por resolución de problemas, es una estrategia prometedora para contribuir con la evolución de competencias científicas apoyadas por la evidencia de cambios conceptuales (saber), actitudinales (saber hacer, saber cómo y querer hacer) $y$ procedimentales (hacer saber y poder hacer) en los estudiantes. La promoción de éstas habilidades favorecerán, desde la educación científica, la formación de personas que actúen armónicamente como seres sociales comprometidos con sus entornos naturales y culturales (saber ser).

\section{Bibliografía}

- Adúriz-Bravo, A. e Izquierdo, M. (2002), "Acerca de la didáctica de las ciencias como disciplina autónoma”, en Revista Electrónica de Enseñanza de las Ciencias [en línea], vol. 1, núm. 3., disponible en: http://www.saum.uvigo.es/reec, recuperado:

- Ariza, L. y Currea, M. (2004), Una Visión de los Contenidos Actitudinales en el Marco de la Enseñanza y Aprendizaje de la Química en Estudiantes de Educación Media [trabajo de grado], Bogotá, Universidad Distrital Francisco José de Caldas, Licenciatura en Química.

— Ausubel, D.; Novak, J. y Hanassian, H (1976), Psicología Educativa, un punto de vista cognoscitivo, México D.F., Editorial Trillas.

- Benarroch, A. (2001), "Una interpretación del desarrollo cognoscitivo de los alumnos en el área de la naturaleza corpuscular de la materia”, en Enseñanza de las Ciencias, vol. 19, núm. 1, pp.123-134.

- De Posada, J. (1999), "Concepciones de los alumnos sobre el enlace químico antes, durante y después de la enseñanza formal", en Enseñanza de las Ciencias, vol. 17, núm. 2, pp. 227-245

- Estany, A. (1990), Modelos de Cambio Científico, Barcelona, Editorial Crítica.

- Fourez, G. (1994), La construcción del conocimiento cientifico, Madrid, Ediciones de Narcea S.A.

- Novak, J. (1988), "Constructivismo humano: un consenso emergente", en Enseñanza de las Ciencias, vol. 6, núm. 3, pp. 213-223.

- Novak, J. y Gowin, D. (1988), Aprendiendo a aprender, Barcelona, Ediciones Martínez Roca. 
- Pozo, J. et. ál (1991), Procesos cognitivos en la comprensión de la ciencia: las ideas de los adolescentes sobre la Química, Madrid, Centro de publicaciones del Ministerio de Educación y Ciencia.

- Pozo, J. (1999), "Sobre las relaciones entre el conocimiento cotidiano de los alumnos y el conocimiento científico: del Cambio Concep- tual a la Integración Jerárquica" en Enseñanza de las ciencias, núm. extra.

- Quintanilla, M. (2005), "Historia de la ciencia y formación del profesorado: una necesidad irreductible", en Revista de la Facultad de Ciencia y Tecnología, Investigación en Experiencias Didácticas en Matemáticas, Ciencias Experimentales y Tecnologías, núm. extra.

\section{ANEXOS No. 1}

\section{RED DE PREGUNTAS}

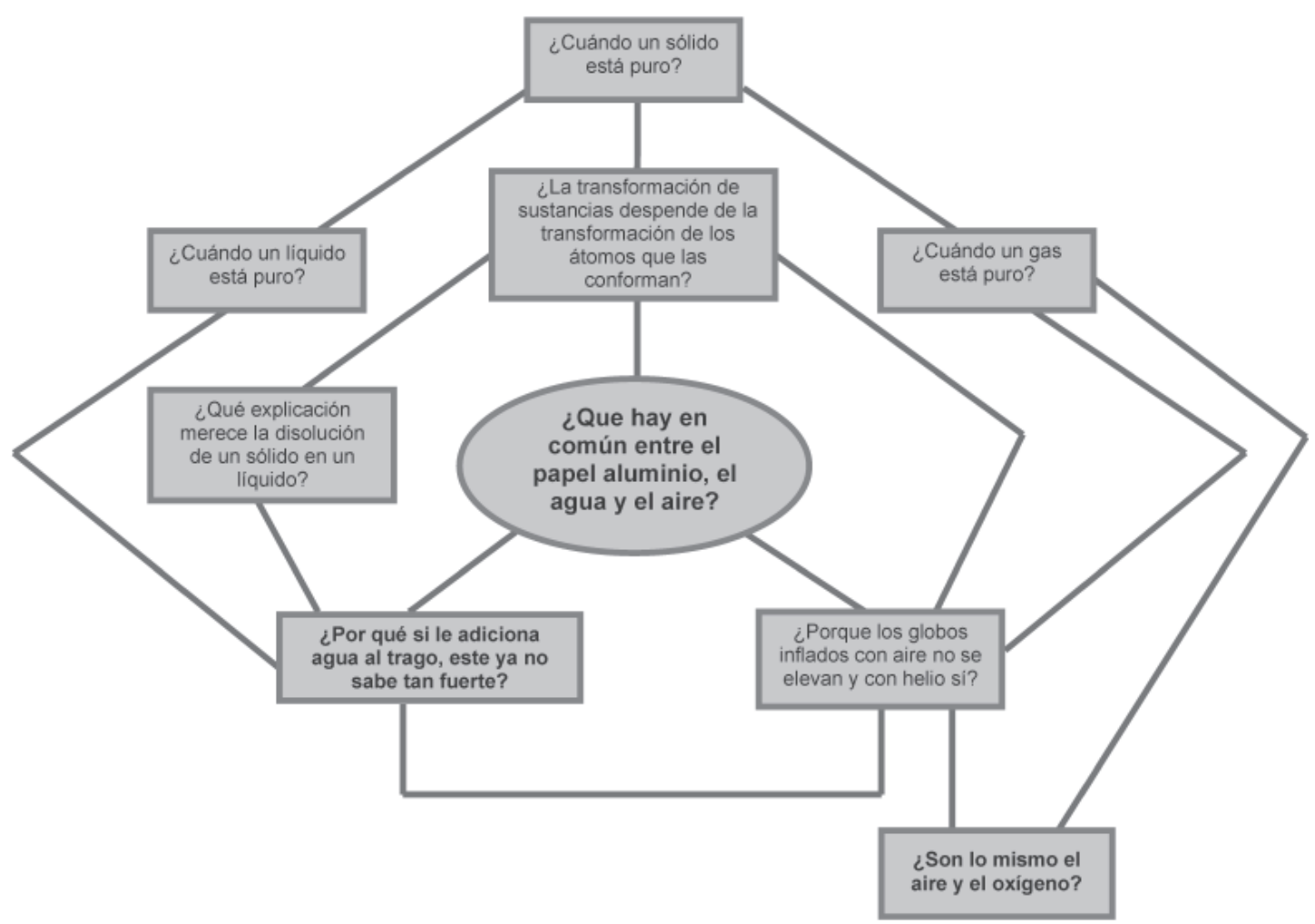


2. MAPA HISTÓRICO

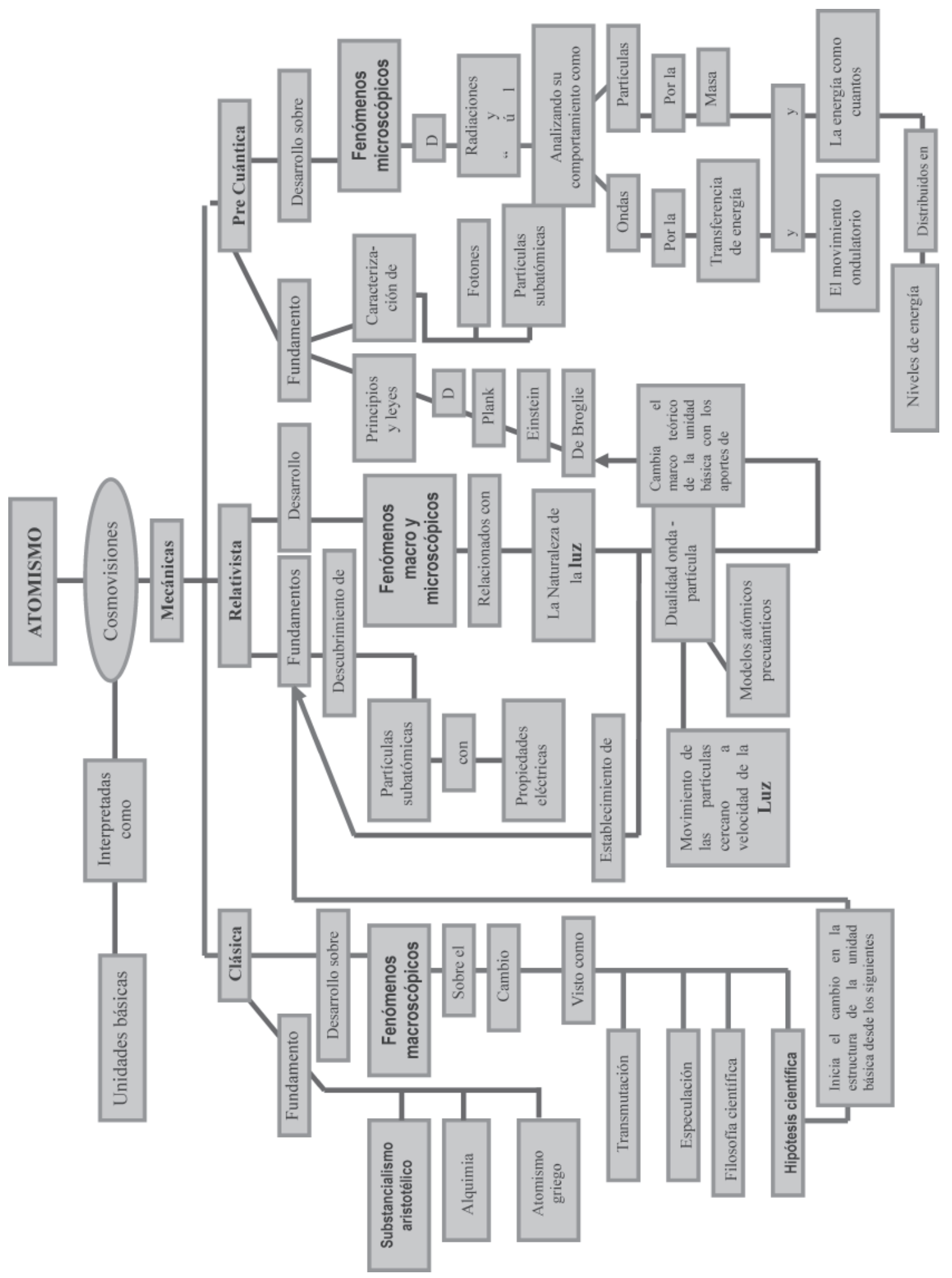

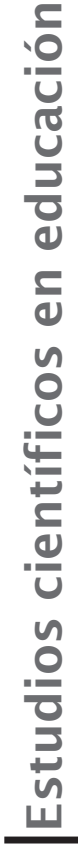

REVISTA CIENTÍFICA / ENERO -DICIEMBRE DE 2010 / No. 12 / BOGOTÁ, D.C. 